\title{
Temperature-Limited Floral Longevity in the Large-Flowered Mediterranean Shrub Cistus Ladanifer (Cistaceae)
}

ARTICLE in INTERNATIONAL JOURNAL OF PLANT SCIENCES · FEBRUARY 2015

2 AUTHORS:

\section{Alberto L Teixido}

Federal University of Minas Gerais

27 PUBLICATIONS 50 CITATIONS

SEE PROFILE
Fernando Valladares

The National Museum of Natural Sciences

293 PUBLICATIONS 8,495 CITATIONS

SEE PROFILE 


\title{
TEMPERATURE-LIMITED FLORAL LONGEVITY IN THE LARGE-FLOWERED MEDITERRANEAN SHRUB CISTUS LADANIFER (CISTACEAE)
}

\author{
Alberto L. Teixido ${ }^{1, *}$ and Fernando Valladares*' $\dagger$ \\ *Área de Biodiversidad y Conservación, Escuela Superior de Ciencias Experimentales y Tecnología, Universidad Rey Juan Carlos, Tulipán s/n, \\ Móstoles, E-28933 Madrid, Spain; and +Museo Nacional de Ciencias Naturales, Consejo Superior de Investigaciones Científicas, \\ Serrano 115 dpdo, E-28006 Madrid, Spain
}

Editor: Christina Caruso

Premise of research. Larger and longer-lived flowers are more frequently pollinated, but they are associated with high maintenance costs due to greater water usage. The Mediterranean climate limits plant reproduction and maximizes resource use efficiency, so smaller and short-lived flowers are potentially advantageous under hot and dry conditions.

Methodology. We experimentally evaluated the relationship between the floral longevity of Cistus ladanifer, a large-flowered Mediterranean shrub, and pollination, flower size, and temperature at two different altitudes with contrasting climatic conditions. We hypothesized that flower size and longevity would be reduced in the drier and hotter conditions of the lower-altitude site. In addition, we expected that floral longevity would decrease with experimental pollen deposition, flower size, and temperature. We pollinated and capped flowers to evaluate the variation in floral longevity by comparing actual and potential floral longevity at low and high altitudes.

Pivotal results. In agreement with our hypothesis, flower size was observed to increase with altitude, but, contrary to expectations, floral longevity decreased at the high altitude since temperature increased during the flowering time. Floral longevity tended to be short but depended on pollination, such that potential floral longevity was greater than actual floral longevity. Capped flowers lasted longer $(1.31 \pm 0.65 \mathrm{~d})$ than control $(0.95 \pm 0.39 \mathrm{~d})$ or hand-pollinated $(0.60 \pm 0.08 \mathrm{~d})$ flowers. At the high altitude, larger flowers and higher temperatures resulted in a reduction in both the observed and potential floral longevity, but temperature had a differential effect in limiting potential floral longevity.

Conclusions. Our experimental survey reported short-lived flowers and limitations in floral longevity when flowers were larger and temperatures were higher. Overall, the results reveal an important effect of temperature on increasing floral maintenance costs, imposing selective pressures on flower size, and longevity in large-flowered Mediterranean plants.

Keywords: actual floral longevity, altitude, floral maintenance costs, flower size, pollen deposition, potential floral longevity.

Online enhancements: appendix tables.

\section{Introduction}

Floral longevity, the length of time that flowers remain open and functional, favors the processes of pollen removal and deposition on the flower (Primack 1985; Ashman and Schoen 1994). Although longer-lived flowers increase the opportunity for reproductive success, floral longevity also entails costs (Ashman and Schoen 1997; Castro et al. 2008). The evolution of floral longevity is thought to be shaped by natural selection to

\footnotetext{
1 Author for correspondence; e-mail: alberto.teixido@urjc.es.
}

Manuscript received July 2014; revised manuscript received October 2014; electronically published December 19, 2014. maximize pollen transfer while minimizing floral maintenance costs (Ashman and Schoen 1994, 1996). For example, longer flower life spans have been reported to be associated with infrequent pollination, while a reduction in floral longevity is induced by pollination (van Doorn 1997; Sargent and Roitberg 2000; Giblin 2005; Weber and Goodwillie 2013). Likewise, floral longevity increases with higher altitude and greater water availability and/or under cooler temperatures (Yasaka et al. 1998; Arathi et al. 2002; Vesprini and Pacini 2005; Arroyo et al. 2013; Jorgensen and Arathi 2013).

The vast majority of studies examining variations in floral longevity have focused on alpine ecosystems in several different regions of the world, which tend to have a low frequency of pollinators under unsuitable weather conditions (e.g., Blionis 
and Vokou 2001; Giblin 2005; Steinacher and Wagner 2010; Arroyo et al. 2013). In these stochastic unpredictable environments, temperature-driven potential floral longevity can be prolonged to assure reproduction when pollinators are absent, which contrasts with the shorter actual floral longevity that has been measured in pollinated flowers (Steinacher and Wagner 2010; Arroyo et al. 2013). Other than such studies, many determinants of variation in floral longevity remain poorly understood, particularly those associated with hot and dry ecosystems. Under such conditions, transpirational cooling is critical for maintaining plant organs at an optimal physiological temperature (Erickson and Markhart 2002; Galen 2005). Thus, water shortage can prevent the normal development of flowers, affecting both fruit and seed production (Galen 2005; Fang et al. 2010). If temperature increases pollinator visitation, an early and efficient pollination would shorten actual floral longevity in self-incompatible species or those with low reproductive assurance, thus allowing a higher resource allocation to develop seeds. However, if late pollination occurs, a prolonged floral longevity could reduce such a resource allocation to develop seeds (Ashman and Schoen 1997; Ishii and Sakai 2000; Castro et al. 2008). In this context, an optimal regulation of floral longevity may buffer costs of delaying fertilization by means of a fast fitness accrual, a scenario in which short-lived flowers should predominate in these environments (Primack 1985; Ashman and Schoen 1994; Galen 2005).

In addition, two other aspects of ephemeral flower life span remain understudied. First, although flower size is known to influence floral longevity (Stratton 1989; van Doorn and Schröder 1995; Arroyo et al. 2013), much remains to be learned about the relationship between floral longevity and flower size variation in a given species. Floral longevity may increase with size among different populations where investment in producing flowers is high and pollination probability is low. However, a negative correlation would be expected within populations based on the arguments used to explain floral longevity as a resource allocation strategy, as larger flowers require more resources for maintenance and pollinator attraction (Ashman and Schoen 1994, 1996). Second, it is unclear whether flower size, temperature, and/or precipitation differentially affect floral longevity, even in absence of pollination, and what the net outcome would be in a population with the hottest and driest conditions and the smallest flowers.

A model scenario to examine those understudied determinants of variation in floral longevity and to evaluate whether fast fitness accrual rates entail short-lived flowers under hot and dry conditions is the Mediterranean region. High temperatures and water shortage over the summer generally characterize the climate in this region (Blondel and Aronson 1999; Quézel and Médail 2003; Thompson 2005). These harsh conditions strongly constrain plant survival and reproduction (Larcher 2000; Thompson 2005; Aragón et al. 2008). Phenologically and morphophysiologically conservative resource use strategies and water use efficiency appear to be adaptative and to play a key role in these environments (Joffre et al. 1999; Valladares et al. 2000, 2008; Thompson 2005). A fundamental question in Mediterranean ecology is how plants tolerate these stressful conditions and are able to flower and fruit. For example, a reduced flowering period, a delay in floral initiation until the rainy season, and a low investment in nectar reward are common phenomena in many Mediterranean plants (e.g., Herrera 1985; Verdú et al. 2002; Aragón et al. 2008). In highly self-incompatible entomophilous plants, reproduction requires that pollinators are attracted by a flower, and so flower size is an important trait in their reproductive ecology. Larger flowers in the Mediterranean zone have higher pollinator visit rates and greater reproductive success (e.g., Herrera 1996; Thompson 2001; Arista and Ortiz 2007; Barrio and Teixido 2014). However, the benefits resulting from the attractiveness of larger flowers can be offset by greater resource costs associated with the increases in water use for floral development and maintenance (Galen et al. 1999; Elle and Hare 2002; Teixido and Valladares 2014). As a consequence, Mediterranean environments may constrain flower production, making small flowers potentially advantageous. As an alternative explanation to selective pressures, phenotypic plasticity in flower size may also result in reduced forms in hotter and drier sites (Barrett et al. 2004; Herrera 2005).

Despite such assumptions, several common Mediterranean species such as rockroses (Cistus spp.) exhibit large flowers. These diurnal-pollinated and spring-flowering shrubs experience elevated floral physiological costs in terms of water and carbon, especially larger-flowered species under high temperatures (Teixido and Valladares 2014). A direct investment of these resources in floral attractiveness is accompanied by indirect costs in terms of fruit and seed production (Teixido and Valladares 2013). In this regard, elevated costs of flower size may involve low margins of variation in floral longevity and counteract the benefits associated with pollen transfer. Therefore, potential floral longevity in the absence of pollination may also be similar to actual floral longevity. This idea would be congruent with a predominance of short-lived flowers under hot and dry environments, in agreement with a fast fitness accrual model.

In this study, we evaluated the combined effects of pollination, flower size, and temperature on floral longevity in Cistus ladanifer, a large-flowered, generalist, and self-incompatible Mediterranean shrub (Talavera et al. 1993). This species occurs along an altitudinal range with significant differences in flower size, ranging from 5 to $11 \mathrm{~cm}$ in diameter (Teixido et al. 2011). Experimentally, we assessed the longevity of pollination-excluded, unmanipulated, and hand-pollinated flowers from two different altitudes. The altitudinal study was intended to reflect differences in flower size and temperature, and higher altitude was expected to be associated with lower temperature and larger flowers. Altitude may also be related to differences in the pollination environment affecting floral longevity. Although we did not record pollinator visitation in this study, we observed that visit rates were constant and that differences in the composition of pollinators do not affect pollen limitation or female fitness between altitudes (Barrio and Teixido 2014). We hypothesized that (1) flower size and longevity are reduced under drier and hotter conditions (i.e., at the lower altitude); (2) floral longevity decreases with experimental pollen deposition and increases when pollination is prevented, such that actual floral longevity is shorter than potential floral longevity at both altitudes; (3) for all treatments and at each altitude, floral longevity decreases with flower size and temperature; and (4) temperature differentially affects floral longevity, such that higher temperatures may 
limit this trait independent of pollination, thus matching potential with actual floral longevity.

\section{Material and Methods}

\section{Species and Study Area}

Cistus ladanifer (Cistaceae) is a shrub $100-250 \mathrm{~cm}$ in height that inhabits acid and dry soils in warm open areas of the western Mediterranean region. Flowering phenology spans from March to June, and each plant produces white flowers, often exhibiting dark-colored spots at their bases. The flowers of this species are the largest in the family and are homogamous, polliniferous, and self-incompatible; secrete some nectar (Herrera 1992; Talavera et al. 1993); and are mainly pollinated by bees, beetles, and flies (Talavera et al. 1993). Flower opening occurs synchronously at dawn each day. The study was conducted from April to June of 2009 at two different altitudes (high and low) in the Madrid province, central Spain (lat. $39^{\circ} 53^{\prime}-41^{\circ} 09^{\prime} \mathrm{N}$, long. $3^{\circ} 03^{\prime}-4^{\circ} 34^{\prime} \mathrm{W}$ ). These altitudes exhibited clear differences in air temperature and humidity. The experimental design to study flower size and longevity (table 1) involved three replicate populations at each of the two altitudes. All populations had a similar orientation (south), slope $\left(0^{\circ}-10^{\circ}\right)$, and tree canopy cover $(0 \%-$ $10 \%)$. Sunny and southern slopes were chosen to remove environmental effects as much as possible. We note that the sympatric species Cistus laurifolius, which is found at the high-altitude site, does not coflower or overlap with the flowering of our study species.

\section{Floral Longevity Response}

A total of 10 similar plants $>1 \mathrm{~m}$ high was randomly selected from each population and tagged during the flowering peak (approximately $2 \mathrm{wk}$ ) in a $20 \times 20-\mathrm{m}$ plot. All plants were at a similar phenological stage when sampled, and there was no overlap between altitudes at flowering time. Air temperature $\left({ }^{\circ} \mathrm{C}\right)$, air relative humidity $(\%)$, soil moisture $(\%)$, and solar irradiance $\left(\mu \mathrm{mol}\right.$ PAR $\left.\mathrm{m}^{-2} \mathrm{~s}^{-1}\right)$ were recorded at the site of each population. Sensors were used for air temperature and relative humidity (Hobo H08-032-08 datalogger; Onset, Pocasset, MA) located $1 \mathrm{~m}$ above the ground. Readings from each sensor were recorded every $30 \mathrm{~min}$ over the period of study. Soil moisture was recorded at 3-5 points below the canopy in the understory of each plant, and sensors were calibrated based on integrated TDR technology $\left(\mathrm{ECH}_{2} \mathrm{O}\right.$ EC20; Decagon Devices, Pullman, WA). Solar irradiance was recorded every $30 \mathrm{~min}$ with a quantum sensor (QSO-SUN; Apogee, Logan, UT). All microclimatic variables were significantly correlated with each other between the two altitudes (tables A1, A2, available online). Air temperature was positively correlated with solar irradiance and negatively correlated with air relative humidity and soil moisture. As a consequence, we used only air temperature as a predictor variable to explain variation in floral longevity. We then recorded mean air temperatures at each altitude during the respective sampling time and related floral longevity to daily mean air temperature.

We also evaluated the relationship between variation in floral longevity and pollination by conducting three different treatments, each using 12 randomly selected individual flowers per plant: hand-pollinated flowers with xenogamous pollen, pollination-excluded flowers by capping of stigmas (hereafter termed capped flowers), and unmanipulated naturally pollinated control flowers. Hand-pollinated and control flowers were used to assess actual floral longevity, whereas capped flowers were used to determine potential floral longevity. Hand pollination was carried out with a fresh pollen mixture collected from 10 different individuals within a $10-\mathrm{m}$ radius of the recipient flower (Giblin 2005; Castro et al. 2008). Using a paintbrush, we then deposited outcross pollen on the stigma $2 \mathrm{~h}$ after anthesis to ensure stigmatic receptivity (Herrera 1992) until the stigmatic area was fully covered. Capping was carried out after flowers were totally open to avoid damage. We note that capping took place early in the morning and, subsequently, flowers did not receive any pollinator visits, so no pollen was deposited on stigma. The caps, which were made of straw and sellotape, were attached to

Table 1

Location and Ecological Data Related to the Cistus ladanifer Experimental Study Sites

\begin{tabular}{|c|c|c|c|c|}
\hline Study sites & $\begin{array}{l}\text { Altitude } \\
(\mathrm{m})\end{array}$ & Climate & Substrate & Vegetation cover \\
\hline \multicolumn{5}{|l|}{ Lower-altitude sites: } \\
\hline $\begin{array}{l}\text { Monte del Pardo (lat. } 40^{\circ} 34^{\prime} \mathrm{N} \text {, long. } \\
3^{\circ} 42^{\prime} \mathrm{W} \text { ) }\end{array}$ & 732 & Dry $533 \mathrm{~mm}, 14^{\circ} \mathrm{C}$ & $\begin{array}{l}\text { Clay and } \\
\text { sand }\end{array}$ & $\begin{array}{l}\text { Dehesa with Quercus ilex and Pinus pinea interspersed } \\
\text { in a shrub matrix }\end{array}$ \\
\hline $\begin{array}{l}\text { Monte del Pardo (lat. } 40^{\circ} 33^{\prime} \mathrm{N} \text {, long. } \\
3^{\circ} 42^{\prime} \mathrm{W} \text { ) }\end{array}$ & 741 & Dry $529 \mathrm{~mm}, 14^{\circ} \mathrm{C}$ & $\begin{array}{l}\text { Clay and } \\
\text { sand }\end{array}$ & $\begin{array}{l}\text { Dehesa with } Q \text {. ilex and } P \text {. pinea interspersed in a } \\
\text { shrub matrix }\end{array}$ \\
\hline $\begin{array}{l}\text { Monte Valdelatas (lat. } 40^{\circ} 32^{\prime} \mathrm{N} \text {, } \\
\text { long. } 3^{\circ} 41^{\prime} \mathrm{W} \text { ) }\end{array}$ & 725 & Dry $520 \mathrm{~mm}, 14^{\circ} \mathrm{C}$ & $\begin{array}{l}\text { Clay and } \\
\text { sand }\end{array}$ & $\begin{array}{l}\text { Dehesa with } Q \text {. ilex and } P \text {. pinea interspersed in a } \\
\text { shrub matrix }\end{array}$ \\
\hline \multicolumn{5}{|l|}{ Higher-altitude sites: } \\
\hline $\begin{array}{l}\text { Becerril de la Sierra (lat. } 40^{\circ} 43^{\prime} \mathrm{N} \text {, } \\
\left.\text { long. } 3^{\circ} 58^{\prime} \mathrm{W}\right)\end{array}$ & 1163 & $\begin{array}{l}\text { Subhumid } 844 \mathrm{~mm} \text {, } \\
\quad 11^{\circ} \mathrm{C}\end{array}$ & Granite & $\begin{array}{l}\text { Patchy scrubland with Cistus laurifolius and Juniperus } \\
\text { oxycedrus }\end{array}$ \\
\hline $\begin{array}{l}\text { Navacerrada (lat. } 40^{\circ} 43^{\prime} \mathrm{N} \text {, long. } \\
4^{\circ} 00^{\prime} \mathrm{W} \text { ) }\end{array}$ & 1176 & $\begin{array}{l}\text { Subhumid } 878 \mathrm{~mm} \text {, } \\
11^{\circ} \mathrm{C}\end{array}$ & Granite & Patchy scrubland with C. laurifolius and J. oxycedrus \\
\hline $\begin{array}{l}\text { Navacerrada (lat. } 40^{\circ} 43^{\prime} \mathrm{N} \text {, long. } \\
4^{\circ} 00^{\prime} \mathrm{W} \text { ) }\end{array}$ & 1199 & $\begin{array}{l}\text { Subhumid } 878 \mathrm{~mm} \text {, } \\
\quad 11^{\circ} \mathrm{C}\end{array}$ & Granite & Patchy scrubland with C. laurifolius and J. oxycedrus \\
\hline
\end{tabular}

Note. Climate column shows the annual mean rainfall $(\mathrm{mm})$ and the annual mean temperature $\left({ }^{\circ} \mathrm{C}\right.$; Ninyerola et al. $\left.2005 ; n=20 \mathrm{yr}\right)$. 
cover the stigma. Flowers were not bagged to avoid elevated floral transpiration rates that might affect floral longevity. The presence of the caps did not alter visitation of the flowers by pollinators (A. L. Teixido, personal observation). Thus, flowers were visited by pollinators, and pollen removal might also affect the potential floral longevity (e.g., Ishii and Sakai 2000). We have taken this assumption into consideration in our discussion.

Treatments were conducted between April 20 and May 3 at the low-altitude site and between May 26 and June 8 at the high-altitude site. Capping was carried out between 0700 and 0900 hours, before pollinator activity and any pollen deposition on stigmas, and hand pollination was carried out between 0900 and 1100 hours. Overall, we selected and tagged 36 flowers per plant, 12 per treatment, giving a sample size of 1080 flowers per altitude (total $n=2160$ flowers). We also measured the diameter of the corolla (centimeter to the nearest millimeter) using a ruler as a proxy of flower size to examine its relationship with floral longevity. Corolla diameter $(\mathrm{cm})$ and flower area $\left(\mathrm{cm}^{2}\right)$ are strongly correlated in the study species (Teixido and Valladares 2014). When a flower remained open for several days, the average diameter was used in the analysis.

To record floral longevity, we monitored flowers once between 1400 and 1500 hours and again between 1900 and 2000 hours each day. Pollination occurs diurnally, and those flowers that remain open between 1900 and 2000 hours start closing and reopen at dawn the next morning. Thus, floral longevity was recorded as a fraction of days according to the following criteria: $0.5 \mathrm{~d}$ for petals falling off before 1400 or 1500 hours of the first day, $1 \mathrm{~d}$ when falling off after 1500 hours but before 1900 or 2000 hours of the first day, $1.5 \mathrm{~d}$ for petals falling off before 1400 or 1500 hours of the second day, and so on. This protocol thus allowed a determination of the flower longevities based on the fraction of the day. As a general rule, during warm and sunny days that were suitable for pollinator activity, flowers lasted until midday, and only under cloudy, cold, and/or rainy days did flowers have an extended life span during the afternoon and/or following days.

\section{Statistical Analysis}

We used two different generalized linear mixed models (GLMMs) to model the variability of flower size as a function of altitude (fixed factor) and the variability of floral longevity as a function of altitude and treatment (fixed factors). In the first GLMM, modeling the variability of flower size, we included population within an altitude and plant within a population as random factors. In the second GLMM, modeling the variability of floral longevity, we included population within an altitude and plant within a population as random factors, flower size and temperature as covariates, and the interactions altitude $x$ treatment, flower size $\times$ altitude, flower size $\times$ treatment, temperature $\times$ altitude, and temperature $\times$ treatment. A significant altitude $x$ treatment outcome indicates a different effect of treatments on floral longevity between altitudes, whereas the other interactions indicate differences in regression slopes between floral longevity with flower size and/or temperature between altitudes and/or treatments. We assumed a normal error distribution with an identity link function for flower size and a Poisson error distribution with a logit link function for floral longevity. The assumptions of normality and homogeneity of variance were tested using Shapiro-Wilk's and Levene's tests, respectively. For all models, we used the restricted maximum likelihood test, and to assess significant differences among treatments at each altitude for floral longevity, their means were compared using post hoc HSD (honest significant distance) tests. All the computations were performed using the GLIMMIX macro function of SAS (SAS statistical package; SAS Institute, Cary, NC).

For the GLMMs, the response variable, which is a (link) function from the original data, is a linear combination of fixed and random factors (Littell et al. 1999). Since our data set had a nested structure and our experimental units (flowers) were not fully independent, we considered variation within plants by including plant within population as a random factor in our model. In this way, we included a statistical control for this source of variation. Since our model is mixed, by including the term plant nested in population, we partition out this effect and can then focus on the variation of interest-variation in altitude, treatment, flower size, and temperature-and the interactions between altitude and treatment, altitude and flower size, altitude and temperature, treatment and flower size, and treatment and temperature. Additionally, since the assignment of the number of degrees of freedom of residuals is critical when using a complex experimental design, we followed Satterthwaite's method to determine the number of degrees of freedom of residuals (Littell et al. 1996; Quinn and Keough 2002).

Given the reported importance of flower size and temperature, we specifically examined how these factors influenced floral longevity. For flower size, we correlated individual mean flower size with individual mean floral longevity (i.e., mean flower size and longevity per plant) for each treatment. We used a Spearman's rank correlation since our data for floral longevity did not satisfy the assumptions of normality and homogeneity of variance. For temperature, we related mean longevity of flowers measured every day with mean air temperature in the days those flowers remained open for each treatment. We used nonlinear regressions as the best-fitting analysis for our data. The sample size comprised 24 data points for all regressions since we conducted the experiment over a period of $24 \mathrm{~d}$ with $12 \mathrm{~d} /$ altitude.

Although we included the interactions between altitude and treatment with flower size and temperature in our full GLMM modeling of variability in floral longevity, there may have been confounding effects on evaluating the relationship between flower size and temperature and floral longevity since higher temperatures were present at the altitude containing larger flowers (see "Results"). To partition out these possible effects, we subsequently analyzed the specific effects of flower size and temperature for each treatment and altitude separately. Thus, we could address two questions: first, whether temperature (rather than flower size) differentially affected floral longevity, reducing the impact of this trait and matching actual with potential flower life span, and second, whether under similar temperature conditions occurring at each altitude having larger flowers significantly reduced both actual and potential floral longevity. At each altitude, we used Spearman's rank correlations for flower size and nonlinear regressions for temperature, as explained above. All these analyses were performed using STATISTICA v12.0 (Statsoft, Tulsa, OK). 


\section{Results}

\section{Temperature, Flower Size, and Longevity}

Contrary to our expectations, all the measured microclimatic variables corresponded with hotter and drier conditions at the high altitude during the flowering time (table A1). Mean temperature ( $\pm \mathrm{SD}$ ), recorded over $12 \mathrm{~d}$ at each altitude, averaged $14.1^{\circ} \pm 2.8^{\circ} \mathrm{C}$ and $17.2^{\circ} \pm 3.4^{\circ} \mathrm{C}$ at the low and high altitudes, respectively, and this difference was significant (fig. 1A). Cistus ladanifer flower size ranged from 5.9 to $10.8 \mathrm{~cm}$ and was significantly greater (by about $12 \%$ ) at the higher altitude (mean \pm SD: $7.3 \pm 1.3 \mathrm{~cm}$ at the low altitude and $8.2 \pm 1.1 \mathrm{~cm}$ at the high altitude; fig. $1 B$ ). Flower size also differed significantly between populations within each altitude and between plants within populations (table 2). Longevity of individual flowers ranged from 0.5 to
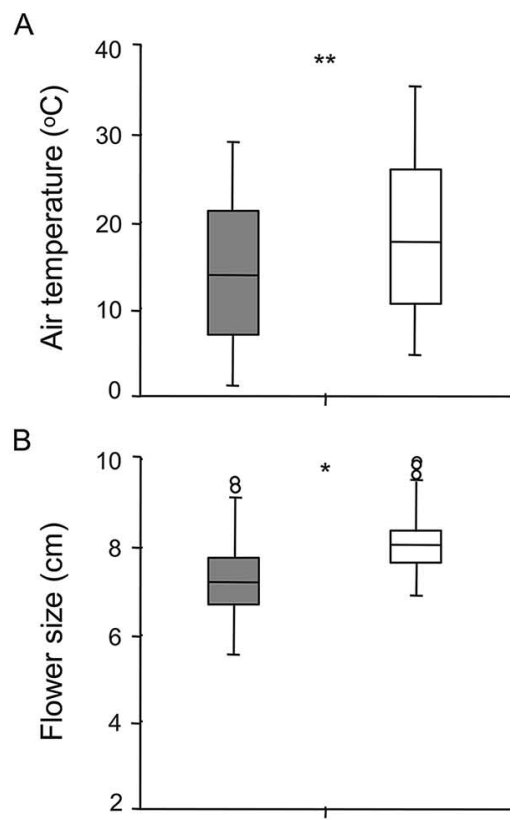

C

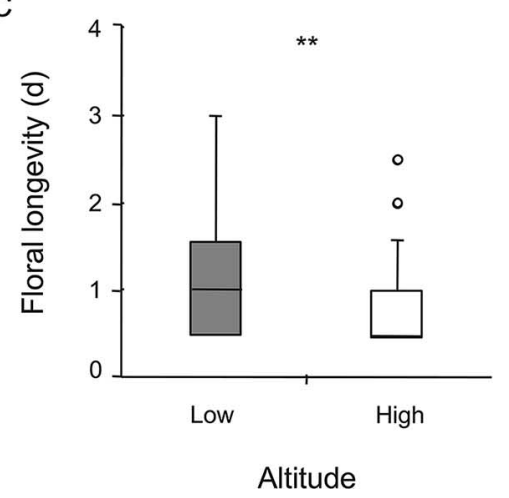

Fig. 1 Box plots of variation in temperature $\left({ }^{\circ} \mathrm{C}\right)$, Cistus ladanifer flower size $(\mathrm{cm})$, and floral longevity $(\mathrm{d})$ at each altitude. Median values are indicated by a horizontal line. One asterisk, $P<0.05$; two asterisks, $P<0.01$.
$3 \mathrm{~d}$, though longevities of $3 \mathrm{~d}$ occurred only in control and capped flowers. Mean actual floral longevity $( \pm S D)$, as measured in control flowers, averaged $1.05 \pm 0.48$ and $0.86 \pm$ $0.35 \mathrm{~d}$ at the low and high altitude, respectively (fig. 1C).

\section{Effects of Altitude and Treatments on Floral Longevity}

Overall, floral longevity differed significantly between altitudes and treatments, flower size, and temperature, as well as the interactions altitude $\times$ treatment and altitude $\times$ temperature, with marginal differences in altitude $\times$ flower size (table 2). For all treatments, flowers at the high altitude lasted for a shorter time than those at the low altitude. At both altitudes, actual floral longevity was shorter than potential floral longevity. Thus, hand-pollinated flowers were the shortest lived and capped flowers the longest lived, whereas control flowers showed intermediate longevities (fig. 2). However, between altitudes, the variation in floral longevity among treatments was significantly variable (altitude $x$ treatment significant; table 2). For example, at the high altitude, potential floral longevity, as measured in capped flowers, was similar to actual floral longevity, as measured in control flowers at the low altitude (i.e., flowers that lasted approximately $1 \mathrm{~d}$; fig. 2 ).

Additionally, the reduction in floral longevity at the high altitude was also apparent through a diminished response of floral longevity. In this regard, capped flowers lasted for $13 \%$ longer and hand-pollinated flowers a $14 \%$ shorter time than control flowers at the high altitude, whereas at the low altitude, capped flowers lasted $31.5 \%$ longer and hand-pollinated flowers a $24 \%$ shorter time than control flowers (fig. 2). Therefore, actual and potential floral longevity were more similar at the high than the low altitude, and potential floral longevity was prolonged under cooler conditions, which was also associated with smaller flowers. The reduction in the variation of floral longevity, as well as the difference between actual and potential floral longevity, was also noticeable across the distribution of the frequency of flower life spans per treatment (fig. 3). Approximately $87 \%$ of the hand-pollinated flowers at the high altitude lasted $0.5 \mathrm{~d}$, while only $80 \%$ did so at the low altitude. For control flowers, $19 \%$ and $32 \%$ lasted $>1 \mathrm{~d}$ at the high or low altitudes, respectively. Last, $69 \%$ of the high-altitude flowers showed a potential floral longevity of $<1 \mathrm{~d}$ and only $14 \%>2 \mathrm{~d}$, compared with $31 \%$ and $35 \%$, respectively, of flowers at the low altitude.

\section{Effects of Flower Size and Temperature on Floral Longevity}

In accordance with the effect of altitude, analyses of the relationship between flower size or temperature and floral longevity revealed that floral longevity significantly decreased with flower size and temperature for all treatments (fig. 4). As reported by our full model, these relationships tended to be constant, independent of treatment (treatment $\times$ flower size and treatment $\times$ temperature were not significant; table 2). We determined that temperature and flower size had different effects on floral longevity. Daily mean temperature showed a nonlinear relationship with flower life span, skewed toward flowers lasting $0.5 \mathrm{~d}$ under high temperatures (approximately $>18^{\circ} \mathrm{C}$ ) independent of pollination, such that potential floral longevity, as measured in capped flowers, was limited and 
Table 2

Generalized Linear Mixed Model (GLMM) of Flower Size and Floral Longevity (2160 Flowers from 60 Plants in 6 Populations at 2 Altitudes)

\begin{tabular}{|c|c|c|c|c|c|c|c|c|}
\hline \multirow[b]{2}{*}{ Effect } & \multicolumn{4}{|c|}{ Flower size } & \multicolumn{4}{|c|}{ Floral longevity } \\
\hline & df & Estimate \pm SD & Test value & $P$ & df & Estimate \pm SD & Test value & $P$ \\
\hline \multicolumn{9}{|l|}{ Random: } \\
\hline Population (altitude) & 5,54 & $1.27 \pm .24$ & 5.21 & .006 & 5,54 & $.01 \pm .00$ & .98 & .163 \\
\hline Plant (population) & 55,2114 & $9.36 \pm 1.17$ & 2.31 & $<\overline{.001}$ & 55,2094 & $.01 \pm .00$ & 1.01 & .159 \\
\hline \multicolumn{9}{|l|}{ Fixed: } \\
\hline Altitude & 1,4 & $1.54 \pm .09$ & 99.32 & $\leq .001$ & 1,4 & $-.28 \pm .04$ & 16.58 & .015 \\
\hline Treatment & $\ldots$ & $\ldots$ & $\ldots$ & $\ldots$ & 2,660 & $-.27 \pm .02$ & 344.55 & $<\overline{.001}$ \\
\hline Altitude $\times$ treatment & $\ldots$ & $\ldots$ & $\ldots$ & $\ldots$ & 2,660 & $-.24 \pm .03$ & 34.96 & $<.001$ \\
\hline Flower size & $\ldots$ & $\ldots$ & $\ldots$ & $\ldots$ & 1,2094 & $-.14 \pm .01$ & 96.32 & $\overline{<.001}$ \\
\hline Temperature & $\ldots$ & $\ldots$ & $\ldots$ & $\ldots$ & 1,2094 & $-.23 \pm .04$ & 209.71 & $\overline{<.001}$ \\
\hline Altitude $\times$ flower size & $\ldots$ & $\ldots$ & $\ldots$ & $\ldots$ & 1,2094 & $-.06 \pm .03$ & 3.57 & .064 \\
\hline Altitude $\times$ temperature & $\ldots$ & $\ldots$ & $\ldots$ & $\ldots$ & 1,2094 & $-.09 \pm .01$ & 4.51 & .021 \\
\hline Treatment $\times$ flower size & $\ldots$ & $\ldots$ & $\ldots$ & $\ldots$ & 1,2094 & $-.04 \pm .03$ & 2.89 & .097 \\
\hline Treatment $\times$ temperature & $\ldots$ & $\ldots$ & $\ldots$ & $\ldots$ & 1,2094 & $-.05 \pm .03$ & 3.30 & .081 \\
\hline
\end{tabular}

Note. Analyses were based on GLMM with normal errors and identity link using restricted maximum likelihood estimation. Random effects were tested with Wald $Z$-tests and the fixed effect with Type III $F$-tests. Significant $P$ values are underlined.

strongly correlated with actual floral longevity (fig. 4A). Otherwise, flower size did not limit potential floral longevity, in contrast with the effects of temperature. Thus, larger flowers showed some variation in floral longevity, and even actual floral longevity, as measured in control flowers, did not correlate with the actual floral longevity of hand-pollinated flowers (fig. 4B).

Our analyses also partially distinguished the possible confounding effects of flower size and temperature due to the presence of both larger flowers and higher temperatures at the high altitude. Moreover, these analyses accounted for the interactions between altitude and flower size or temperature. At the low altitude and for the three treatments, flower size

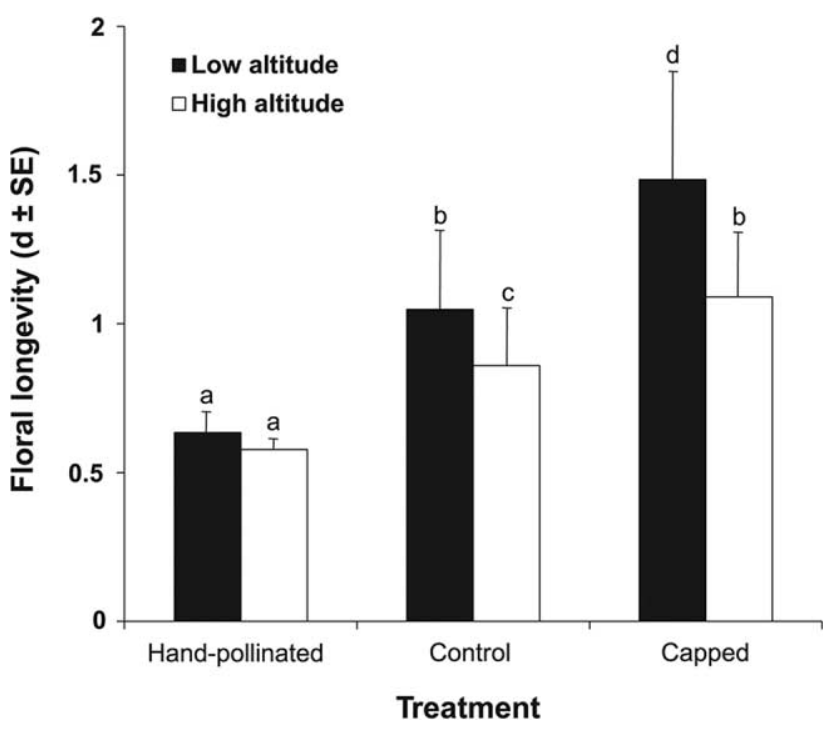

Fig. 2 Differences in Cistus ladanifer floral longevity in the interaction altitude $x$ treatment. Different letters indicate significant differences among treatments using an unequal HSD post hoc test $(P<$ $0.05)$. Columns $=$ mean values; bars $=\mathrm{SE}$. and temperature did not have any effect on floral longevity (tables 3,4). However, at the high altitude, larger flowers and higher temperatures resulted in shorter-lived flowers. Once again, temperature had a stronger effect than flower size on floral longevity, significantly reducing the duration of the flowers in all three treatments (table 3). In contrast, larger flowers reduced the potential floral longevity and actual floral longevity, as measured in control flowers, but did not affect handpollinated flowers (table 4).

\section{Discussion}

Our experimental survey of Cistus ladanifer populations represents an important contribution to understanding how abiotic factors coupled with pollen deposition and flower size can play a role in the amount of time flowers remain open, which has important consequences for plant reproduction. Our data show how altitude has unexpected effects when phenological differences in plants at higher elevations blooming later correspond with higher temperatures compared to lower elevations. We describe here the variation in floral longevity in relation to pollination, flower size, and temperature. Most interestingly, higher temperatures limited floral longevity to less than $1 \mathrm{~d}$, independent of pollination. Overall, our results provide empirical evidence that large flowers tend to be short lived under hot and dry conditions.

However, an important caveat is that we lack data regarding the temporal variation in floral longevity and pollinator visits. Likewise, the correlation reported for the microclimatic variables measured might not be consistent in multiple years. Indeed, differences in the pollination environment may affect floral longevity (Ishii and Sakai 2000; Blionis and Vokou 2001; Arroyo et al. 2013). These differences may also show temporal variation in relation to changes in climatic conditions and ultimately influence floral longevity (Blair and Wolfe 2007). Although we did not record pollinator visitation or any temporal variation in this study, we still report the importance of pollination on variation in floral longevity. Additionally, our results support the assumption for fast fitness accrual and ephemeral 


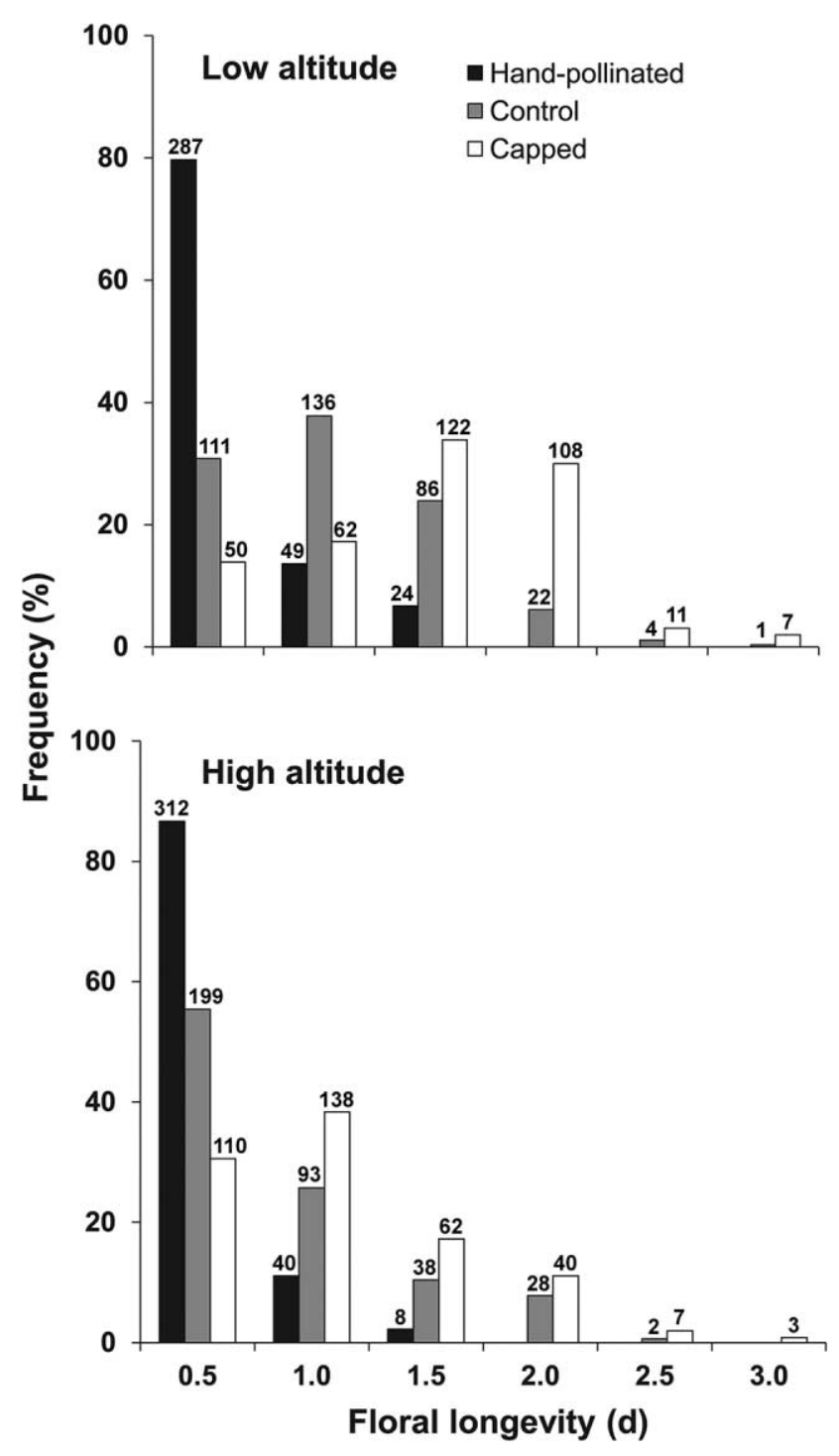

Fig. 3 Frequency (\%) of different Cistus ladanifer floral longevities $(0.5-3.0 \mathrm{~d})$ per treatment and altitude. Absolute numbers of flowers are shown above each bar.

floral longevity in large-flowered plants blooming under a hot and dry environment.

The short floral longevity observed in our study contrasts with floral longevities of several days in Mediterranean ecosystems (Petanidou et al. 1995; Blionis et al. 2001; Berjano et al. 2009; Marques and Draper 2012). However, Petanidou et al. (1995) reported that members of the Cistaceae also show floral longevities of $1 \mathrm{~d}$. Phylogenetic constraints may account for this difference, as several families have flowers that typically last $1 \mathrm{~d}$ (e.g., Commelinaceae, Convolvulaceae, Pontederiaceae [Primack 1985]; Acanthaceae [Endress 1994]; Turneraceae [Arbo 2007]). We propose, however, that there is an alternative plausible explanation to phylogeny in our experimental system: the combined effects of pollination, flower size, and environmental (temperature) influences.
A negative relationship between floral longevity and pollen deposition has been found in many other studies, where pollination of flowers decreased their longevity (Clayton and Aizen 1996; Ishii and Sakai 2000; Arathi et al. 2002; Giblin 2005; Arroyo et al. 2013), while lack of pollinator visits led to extended floral longevity (Ishii and Sakai 2000; Blionis and Vokou 2001; Steinacher and Wagner 2010; Arroyo et al. 2013). This pattern may be related to the importance of female function in resource allocation and the costs of prolonged floral longevity to fruit and seed production (Ashman and Schoen 1997; Castro et al. 2008). Therefore, the limitation of floral longevity to $0.5 \mathrm{~d}$ in pollinated C. ladanifer flowers is in accordance with the elevated floral costs of this
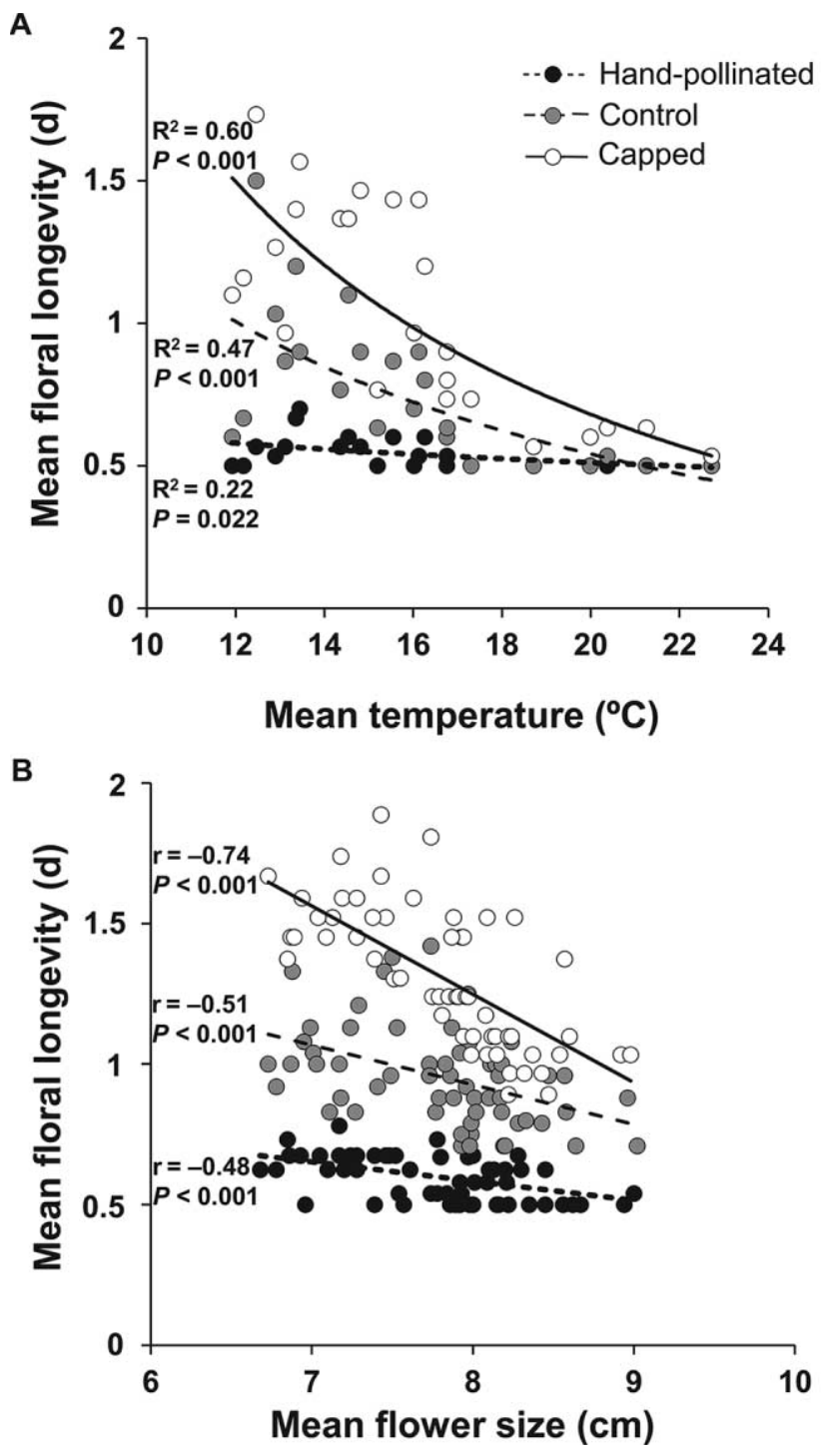

Fig. 4 Differences in Cistus ladanifer floral longevity among treatments at different temperatures $(A)$ and flower sizes $(B)$. Circles denote the mean floral longevity of flowers measured each day versus the daily mean temperature $(A ; n=24 \mathrm{~d} /$ treatment $)$ and of all individuals sampled versus individual mean flower size $(B ; n=60$ individuals). 
Table 3

Linear Regressions of Each Treatment at Each Altitude for Mean Floral Longevity (d) against Mean Temperature $\left({ }^{\circ} \mathrm{C}\right)$

\begin{tabular}{|c|c|c|c|c|c|c|c|c|c|c|c|c|}
\hline \multirow[b]{2}{*}{ Treatment } & \multicolumn{6}{|c|}{ Low altitude } & \multicolumn{6}{|c|}{ High altitude } \\
\hline & $R^{2}$ & $\beta_{0} \pm \mathrm{SD}$ & $\beta_{1} \pm \mathrm{SD}$ & df & $F$ & $P$ & $R^{2}$ & $\beta_{0} \pm \mathrm{SD}$ & $\beta_{1} \pm \mathrm{SD}$ & df & $F$ & $P$ \\
\hline Hand-pollinated & .01 & $1.12 \pm .21$ & $-.00 \pm .02$ & 1,11 & .070 & .797 & .56 & $1.21 \pm .05$ & $-.01 \pm .00$ & 1,11 & 12.885 & .005 \\
\hline Control & .02 & $1.53 \pm .47$ & $-.01 \pm .03$ & 1,11 & .182 & .679 & .60 & $2.50 \pm .35$ & $-.08 \pm .02$ & 1,11 & 14.697 &.$\overline{.003}$ \\
\hline Capped & .06 & $2.20 \pm .55$ & $-.03 \pm .04$ & 1,11 & .581 & .463 & .67 & $2.93 \pm .36$ & $-.09 \pm .02$ & 1,11 & 19.991 & $\overline{.001}$ \\
\hline
\end{tabular}

Note. Significant $P$ values are underlined. $n=12$ data for all regressions.

species, which reduce the fruit and seed production (Teixido and Valladares 2013, 2014). Our data support the general trend of a pollination-mediated reduction in floral longevity and evolution of short-lived flowers by mean fast fitness accrual rates that may minimize floral maintenance costs.

The observed differences in flower life span among treatments and altitudes and their interaction represent an important data set in the context of understanding the ecology and evolution of floral longevity. Altitude may involve differences in the pollination environment that affect floral longevity. Although we did not record pollinator visitation, our pollination treatments were designed to accurately assess the potential and actual floral longevity at both sites. From an evolutionary perspective, variation between actual and potential floral longevity reveals the importance of this trait in the reduction of the balance between maintenance cost and fitness rates (Ashman and Schoen 1994, 1996). At the low altitude, prolonged potential floral longevity may ameliorate slow pollination at reduced maintenance cost rates under cooler temperatures. Likewise, increases in effectiveness and/ or visit rates of pollinators, together with increases in floral maintenance costs, may account for shorter actual floral longevity of open-pollinated control flowers at higher elevation. Consequently, the potential for fitness gain in a short time would exceed the costs associated with floral maintenance (i.e., fast fitness accrual). For example, if we assume identical costs at each altitude, fitness accrual is faster at the high altitude and actual floral longevity decreases since visitation would be higher and/or more effective. Similarly, assuming identical pollinator activity, increased costs at the high altitude would also involve faster fitness accrual as they may exceed potential fitness gain (Ashman and Schoen 1994, 1997). The effects of flower size and temperature (regardless of pollination) on floral longevity that are revealed in our study contribute to distinguishing between these models.

\section{Table 4}

Spearman's Rank Correlations of Each Treatment at Each Altitude for Mean Floral Longevity (d) against Mean Flower Size (cm)

\begin{tabular}{lcll}
\hline Altitude & Hand-pollinated & Control & Capped \\
\hline Low & -.002 & -.020 & -.044 \\
High & -.179 & $\underline{-.368^{*}}$ & $\underline{-.797^{* *}}$ \\
\hline
\end{tabular}

Note. Significant $P$ values are underlined. $n=12$ data for all correlations.

$* P=0.046$.

$\approx * \quad P<0.001$.
Here we provide new insights into the underinvestigated relationship between flower size and longevity. Contrary to our expectations, floral longevity was shorter at the high altitude where flowers were larger. This suggests a negative effect of flower size on floral longevity at a species level, although the potential confounding effects of temperature are likely to be important. We did not detect the patterns of shorter floral longevity in sites with higher temperatures and smaller flowers. We hypothesized that flower longevity would be less at the low altitude despite flowers being smaller, as a consequence of the higher temperature. Given our results, it is difficult to distinguish the effects of flower size and temperature. Acting together, both factors may affect floral longevity and limit its response by reducing potential floral longevity at high altitudes. Alternatively, we expected that, within populations, flower size would reduce floral longevity independent of temperature. This was found to be the case only at the high-altitude site (i.e., under higher temperatures and for larger flowers). One explanation is that smaller-flowered individuals suffered higher pollen limitation levels at this site, thus reducing the amount of pollen deposition on stigmas and prolonging floral longevity. This would be in accordance with the results reported by Barrio and Teixido (2014), where at higher elevations, C. ladanifer flowers were reported to be not only larger but also more pollen limited than at lower sites. Likewise, low levels of pollen limitation at lower altitude may explain the apparent absence of any relationship between flower size and longevity.

Ultimately, our results based on temperature in the absence of pollinator data may explain why both actual floral longevity, as measured in control flowers, and the potential floral longevity of capped flowers were shorter at the high altitude. Interestingly, although inverse relationships between temperature and floral longevity have been reported (Yasaka et al. 1998; Sargent and Roitberg 2000; Giblin 2005; Arroyo et al. 2013), we detected a limited effect on potential floral longevity with higher temperature. Further work is required to elucidate the proximate mechanisms of the patterns reported here, but temperature-mediated increases in transpiration and respiration rates are likely involved (see Teixido and Valladares 2014). In such a scenario, the theoretical expectation of a balance between costs and fitness rates would not be realized. Thus, elevated floral physiological costs may increasingly exceed fitness gain and even disrupt flower function independent of pollination. These factors are important for understanding the ecology and evolution of floral longevity and, specifically, for explaining why large flowers tend to be short lived under hot and dry environments. 
There may be other explanations for the floral longevity relationships found in our study. For example, a relationship between temperature and flower life span may be affected by other correlated factors (e.g., time-related changes in temperature and plant resource status conditioning floral longevity), and biotic factors acting at the individual and/or population level may explain differences in floral longevity. In this context, variation in this trait may be associated with male function, with longer-lived flowers favoring higher amounts of pollen removal (Clayton and Aizen 1996; Ishii and Sakai 2000; Weber and Goodwillie 2007, 2013). If pollinator visitation varied between the two altitudes, this may have influenced potential floral longevity at both sites, although we note that it has been previously reported that pollinator visit rates are similar between altitudes (Barrio and Teixido 2014). Indeed, floral longevity seems to be more related with pollen deposition than with pollen removal (Proctor and Harder 1995; Martini et al. 2003), and our data based on hand-pollinated flowers support this conclusion. Likewise, both the number of displayed flowers and flower position in the inflorescence have been reported as factors that affect floral longevity (Stpiczyńska 2003; Harder and Johnson 2005). It would be interesting to analyze the role of these traits in terms of pollinator service and floral maintenance costs.

In conclusion, our study of C. ladanifer populations provides new insights into the evolution of floral longevity and the combined effects of pollination, flower size, and temperature on this trait. The results showed an altitudinal variation in flower size and floral longevity that was short but not fixed. The main conclusions are that pollen deposition reduces floral longevity and that this response is strongly limited at sites where flowers are larger and temperatures during the flowering time are higher. Together, all these results suggest an important effect of temperature on increasing maintenance costs in large-flowered Mediterranean plants. We propose that further research on floral longevity should focus on sympatric coflowering species with contrasting flower size and patterns of temporal variation beyond patterns recorded among populations. Moreover, it would be interesting to analyze differences in pollinator environments as well as the effects of the floral longevity response on physiological maintenance costs of flowers and, ultimately, on female fitness accrual rates.

\section{Acknowledgments}

We are grateful to two anonymous reviewers for providing helpful comments, and we thank PlantScribe (http://www .plantscribe.com) for editing a final version of the manuscript. We also thank C. Botejara and L. Zamorano for fieldwork support and A. Escudero, B. Martínez, M. Méndez, and S. G. Rabasa for statistical advice. C. Caruso, J. Herrera, and M. Méndez provided valuable comments in early versions of the manuscript. This study was supported by the REMEDINAL project (S-0505/AMB/000355) of Comunidad de Madrid, Spain, and by the CONSOLIDER MONTES project (CSD200800040) of the Spanish Ministerio de Ciencia e Innovación.

\section{Literature Cited}

Aragón CF, A Escudero, F Valladares 2008 Stress-induced dynamic adjustments of reproduction differentially affect fitness components of a semi-arid plant. J Ecol 96:222-229.

Arathi HS, A Rasch, C Cox, JK Kelly 2002 Autogamy and floral longevity in Mimulus guttatus. Int J Plant Sci 163:567-573.

Arbo MM 2007 Turneraceae. Pages 458-466 in K Kubitzki, C Bayer, PF Stevens, eds. The families and genera of vascular plants. Vol 9. Springer, Berlin.

Arista M, PL Ortiz 2007 Differential gender selection on floral size: an experimental approach using Cistus salviifolius. J Ecol 95:973982.

Arroyo MTK, LS Dudley, G Jespersen, DA Pacheco, LA Cavieres 2013 Temperature-driven flower longevity in a high-alpine species of Oxalis influences reproductive assurance. New Phytol 200: 1260-1268.

Ashman T-L, DJ Schoen 1994 How long should flowers live? Nature 371:788-791.

1996 Floral longevity: fitness consequences and resource costs. Pages 112-139 in DG Lloyd, SCH Barrett, eds. Floral biology. Chapman \& Hall, New York.

- 1997 The cost of floral longevity in Clarkia tembloriensis. Evol Ecol 11:289-300.

Barrett SCH, LD Harder, WW Cole 2004 Correlated evolution of floral morphology and mating-type frequencies in a sexually polymorphic plant. Evolution 58:964-975.

Barrio M, AL Teixido 2014 Sex-dependent selection on flower size in a large-flowered Mediterranean species: an experimental approach with Cistus ladanifer. Plant Syst Evol (forthcoming). doi:10.1007/s00606-014-1058-0.

Berjano R, PL Ortiz, M Arista, S Talavera 2009 Pollinators, flowering phenology and floral longevity in two Mediterranean Aristolochia species, with a review of flower visitor records for the genus. Plant Biol 11:6-16.

Blair AC, LM Wolfe 2007 The association between floral longevity and pollen removal, pollen receipt, and fruit production in flame azalea (Rhododendron calendulaceum). Can J Bot 85:414-419.

Blionis GJ, JM Halley, D Vokou 2001 Flowering phenology of Campanula on Mt Olympos, Greece. Ecography 24:696-706.

Blionis GJ, D Vokou 2001 Pollination ecology of Campanula species on Mt Olympos, Greece. Ecography 24:287-297.

Blondel J, J Aronson 1999 Biology and wildlife of the Mediterranean region. Oxford University Press, Oxford.

Castro S, P Silveira, L Navarro 2008 Effects of pollination on floral longevity and costs of delaying fertilization in the out-crossing Polygala vayredae Costa (Polygalaceae). Ann Bot 102:1043-1048.

Clayton S, MA Aizen 1996 Effects of pollinia removal and insertion on flower longevity in Chloraea alpina (Orchidaceae). Evol Ecol 10:653-660.

Elle E, JD Hare 2002 Environmentally induced variation in floral traits affects the mating system in Datura wrightii. Funct Ecol 16:79-88.

Endress PK 1994 Diversity and evolutionary biology of tropical flowers. Cambridge University Press, Cambridge.

Erickson AN, AH Markhart 2002 Flower development stage and organ sensitivity of bell pepper (Capsicum annum L.) to elevated temperature. Plant Cell Environ 25:123-130.

Fang X, NC Turner, G Yan, F Li, KHM Siddique 2010 Flower numbers, pod production, pollen viability, and pistil function are reduced and flower and pod abortion increased in chickpea (Cicer arietinum L.) under terminal drought. J Exp Bot 61:335-345.

Galen C 2005 It never rains but then it pours: the diverse effects of water on flower integrity and function. Pages 75-95 in E Reekie, 
FA Bazzaz, eds. Reproductive allocation in plants. Elsevier, San Diego, CA.

Galen C, RA Sherry, AB Carroll 1999 Are flowers physiological sinks or faucets? costs and correlates of water use by flowers of Polemonium viscosum. Oecologia 118:461-470.

Giblin DE 2005 Variation in floral longevity between populations of Campanula rotundifolia (Campanulaceae) in response to fitness accrual rate manipulation. Am J Bot 92:1714-1722.

Harder LD, SD Johnson 2005 Adaptive plasticity of floral display size in animal-pollinated plants. Proc R Soc B 272:2651-2657.

Herrera CM 1996 Floral traits and plant adaptation to insect pollinators: a devil's advocate approach. Pages 65-87 in DG Lloyd, $\mathrm{SCH}$ Barrett, eds. Floral biology. Chapman \& Hall, New York.

Herrera J 1985 Nectar secretion patterns in southern Spanish Mediterranean scrublands. Isr J Bot 34:47-58.

1992 Flower variation and breeding systems in the Cistaceae. Plant Syst Evol 179:245-255.

2005 Flower size variation in Rosmarinus officinalis: individuals, populations and habitats. Ann Bot 95:431-437.

Ishii HS, S Sakai 2000 Optimal timing of corolla abscission: experimental study on Erythronium japonicum (Liliaceae). Funct Ecol 14:122-128.

Joffre R, S Rambal, C Damesin 1999 Functional attributes in Mediterranean-type ecosystems. Pages 347-380 in FI Pugnaire, F Valladares, eds. Handbook of functional plant ecology. Marcel Dekker, New York.

Jorgensen R, HS Arathi 2013 Floral longevity and autonomous selfing are altered by pollination and water availability in Collinsia heterophylla. Ann Bot 112:821-828.

Larcher W 2000 Temperature stress and survival ability of Mediterranean sclerophyllous plants. Plant Biosyst 134:279-295.

Littell RC, GA Milliken, WW Stroup, RD Wolfinger 1999 SAS system for mixed models. SAS Institute, New York.

Marques I, D Draper 2012 Pollination activity affects selection on floral longevity in the autumnal-flowering plant, Narcissus serotinus L. Botany 90:283-291.

Martini P, C Schlindwein, A Montenegro 2003 Pollination, flower longevity, and reproductive biology of Gongora quinquenervis Ruíz and Pavón (Orchidaceae) in an Atlantic forest fragment of Pernambuco, Brazil. Plant Biol 5:495-503.

Ninyerola M, X Pons, JM Roure 2005 Atlas climático digital de la Península Ibérica: metodología y aplicaciones en bioclimatología y geobotánica. Universidad Autonoma de Barcelona, Barcelona.

Petanidou T, WN Ellis, NS Margaris, D Vokou 1995 Constraints on flowering phenology in a phryganic (East Mediterranean shrub) community. Am J Bot 82:607-620.

Primack RB 1985 Longevity of individual flowers. Annu Rev Ecol Syst 16:15-37.

Proctor HC, LD Harder 1995 Effect of pollination success on floral longevity in the orchid Calypso bulbosa (Orchidaceae). Am J Bot 82:1131-1136.

Quézel P, F Médail 2003 Ecologie et biogéographie des forêts du bassin méditerranéen. Elsevier, Paris.
Quinn G, M Keough 2002 Experimental design and data analysis for biologists. Cambridge University Press, Cambridge.

Sargent RD, BD Roitberg 2000 Seasonal decline in male-phase duration in a protandrous plant: a response to increased mating opportunities? Funct Ecol 14:484-489.

Steinacher G, J Wagner 2010 Flower longevity and duration of pistil receptivity in high mountain plants. Flora 205:376-387.

Stpiczyńska M 2003 Floral longevity and nectar secretion of Platanthera chlorantha (Custer) Rchb. (Orchidaceae). Ann Bot 92:191-197.

Stratton DA 1989 Longevity of individual flowers in a Costa Rican cloud forest: ecological correlates and phylogenetic constraints. Biotropica 21:308-318.

Talavera S, PE Gibbs, J Herrera 1993 Reproductive biology of Cistus ladanifer (Cistaceae). Plant Syst Evol 186:123-134.

Teixido AL, M Méndez, F Valladares 2011 Flower size and longevity influence florivory in the large-flowered shrub Cistus ladanifer. Acta Oecol 37:418-421.

Teixido AL, F Valladares 2013 Large and abundant flowers increase indirect costs of corollas: a study of coflowering sympatric Mediterranean species of contrasting flower size. Oecologia 173:7381.

2014 Disproportionate carbon and water maintenance costs of large corollas in hot Mediterranean ecosystems. Perspect Plant Ecol 16:83-92.

Thompson JD 2001 How do visitation patterns vary among pollinators in relation to floral display and floral design in a generalist pollination system? Oecologia 126:386-394.

2005 Plant evolution in the Mediterranean. Oxford University Press, New York.

Valladares F, E Martínez-Ferri, L Balaguer, E Pérez-Corona, E Manrique 2000 Low leaf-level response to light and nutrients in Mediterranean evergreen oaks: a conservative resource-use strategy? New Phytol 148:79-91.

Valladares F, J Zaragoza-Castells, D Sánchez-Gómez, S Matesanz, B Alonso, A Portsmouth, A Delgado, OK Atkin 2008 Is shade beneficial for Mediterranean shrubs experiencing periods of extreme drought and late-winter frosts? Ann Bot 102:923-933.

van Doorn WG 1997 Effects of pollination on floral attraction and longevity. J Exp Bot 48:1615-1622.

van Doorn WG, C Schröder 1995 The abscission of rose petals. Ann Bot 76:539-544.

Verdú M, J Barrón-Sevilla, A Valiente-Banuet, N Flores-Hernández, P García-Fayos 2002 Mexical phenology: is it similar to Mediterranean communities? Bot J Linn Soc 138:297-303.

Vespirini JL, E Pacini 2005 Temperature-dependent floral longevity in two Helleborous species. Plant Syst Evol 252:63-70.

Weber JJ, C Goodwillie 2007 Timing of self-compatibility, flower longevity, and potential for male outcross success in Leptosiphon jepsonii (Polemoniaceae). Am J Bot 94:1338-1343.

2013 Variation in floral longevity in the genus Leptosiphon: mating system consequences. Plant Biol 15:220-225.

Yasaka M, Y Nishiwaki, Y Konno 1998 Plasticity of flower longevity in Corydalis ambigua. Ecol Res 13:211-216. 